\title{
Sex-related penile fracture with complete urethral rupture: A case report and review of the literature
}

\author{
Marco Garofalo ${ }^{1}$, Lorenzo Bianchi ${ }^{1}$, Giorgio Gentile ${ }^{1}$, Marco Borghesi ${ }^{1}$, Valerio Vagnoni ${ }^{1}$, \\ Hussam Dababneh ${ }^{1}$, Riccardo Schiavina ${ }^{1}$, Alessandro Franceschelli ${ }^{2}$, Daniele Romagnoli ${ }^{1}$, \\ Fulvio Colombo ${ }^{2}$, Beniamino Corcioni ${ }^{3}$, Rita Golfieri ${ }^{3}$, Eugenio Brunocilla ${ }^{1}$ \\ ${ }^{1}$ Department of Urology, University of Bologna, S.Orsola-Malpighi Hospital, Bologna, Italy \\ ${ }^{2}$ Andrology Unit, Sant'Orsola-Malpighi Hospital, Bologna, Italy; \\ ${ }^{3}$ Department of Radiology, University of Bologna, S.Orsola-Malpighi Hospital, Bologna, Italy.
}

\begin{abstract}
Summary Objective: To present the management of a patient with partial disruption of both cavernosal bodies and complete urethral rupture and to propose a non-systematic review of literature about complete urethral rupture.

Material and method - Case report: A 46 years old man presented to our emergency department after a blunt injury of the penis during sexual intercourse. On physical examination there was subcutaneous hematoma extending over the proximal penile shaft with a dorsal-left sided deviation of the penis and urethral bleeding. Ultrasound investigation showed an hematoma in the ventral shaft of the penis with a discontinuity of the tunica albuginea of the right cavernosal corporum. The patient underwent immediate emergency surgery consisted on evacuation of the hematoma, reparation the partial defect of both two cavernosal bodies and end to end suture of the urethra that resulted completely disrupted. Results: The urethral catheter was removed at the 12-th postoperative day without voiding symptoms after a retrograde urethrography. 6 months postoperatively the patients was evaluated with uroflowmetry demonstrating a max flow rate of $22 \mathrm{ml} / \mathrm{s}$ and optimal functional outcomes evaluated with validated questionnaires. 8 months after surgery the patients was evaluated by dynamic magnetic resonance (MRI) of the penis showing only a little curvature on the left side of the penile shaft.

Conclusion: Penile fracture is an extremely uncommon urologic injury with approximately 1331 reported cases in the literature till the years 2001. To best of our knowledge from 2001 up today, 1839 more cases have been reported, only in 159 of them anterior urethral rupture was associated and in only 22 cases a complete urethral rupture was described. In our opinion, in order to prevent long term complications, in case of clinical suspicion of penile fracture, especially if it is associated to urethral disruption, emergency surgery should be the first choice of treatment.
\end{abstract}

KEY WORDS: penile fracture, Complete urethral rupture, Surgical repair.

Submitted 9 February 2015; Accepted 31 July 2015

\section{INTRODUCTION}

Penile fracture is an uncommon urologic injury, with approximately 1331 reported cases in the literature till the year 2001 (1-3). We present the management of a patient with partial disruption of both cavernosal bodies due to sexual intercourse trauma with complete urethral rupture and its surgical reconstruction; we also propose a non-systematic review of literature about penile fracture and anterior urethral rupture.

\section{Case report}

A 46 years old Caucasian man presented in March 2014 to our emergency department 1 hour after blunt injury of the penis during sexual intercourse. The patient reported a cracking sound and severe penile pain followed by immediate detumescence after a blunt trauma against his partner's perineum during sexual intercourse. He didn't notice blood at the urethral meatus but he was not able to void. On physical examination there was swelling and subcutaneous hematoma extending over the proximal penile shaft, the scrotum and the pubis with a dorsal-left sided deviation of the penis. The typical "rolling sign" was present in the left side of penis' shaft (4 cm from the base of the penis). The scrotum was swollen and painful on physical examination and the testicles were palpable with difficulty due to the oedema. Abdominal examinations showed bloating of the lower abdomen: due to inability to void, a 14 Ch Foley catheter was easily insert with emission of clear urine. The patient was firstly managed with conservative therapy: ice pack on the penis, fluid infusion and antibiotic prophylaxis. Penile ultrasound (US) showed the presence of hematoma in the ventral shaft of the penis with a rupture of the tunica albuginea of the right cavernosal corpora and oedema of the subcutaneous tissue. One hour later finding of blood at the urethral meatus suggested an urethral injury and the blood exams revealed anemia. We informed the patients about the potential postoperative complications such as necrosis, stricture, penile deformity or shortening and the potential complication not to operate; after having his consent he underwent immediate emergency surgery. Under spinal anesthesia, after a penile degloving a partial rupture of both cavernosal bodies was identified. After the evacuation of the hematoma in the ventral shaft of the penis, a complete urethral disruption and partial bilateral cavernosal bod- 
Figure 1.

Intraoperative photo demonstrating bilateral partial rupture of the corpora cavernosa and complete rupture of corpum spongiosum.

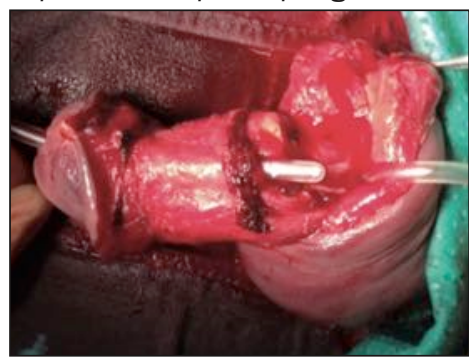

Figure 2.

Intraoperative photo demonstrating end-to-end anastomosis in one layer of the urethra and suture of both corpora cavernosa,

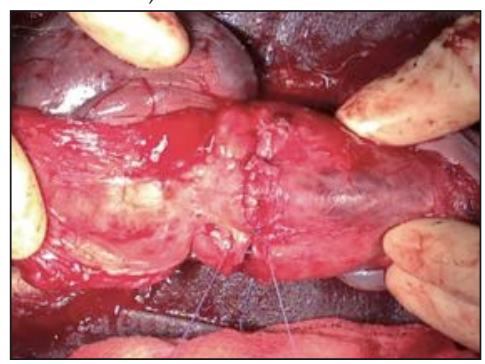

Figure 3.

Postoperative retrograde urethrogram (12 days postoperatively)

demonstrating absence of urethral stricture.

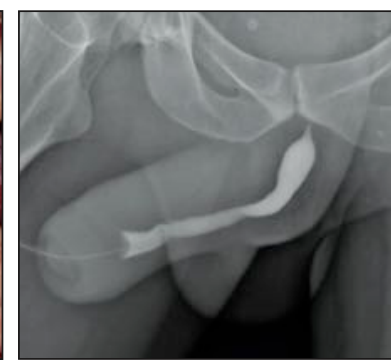

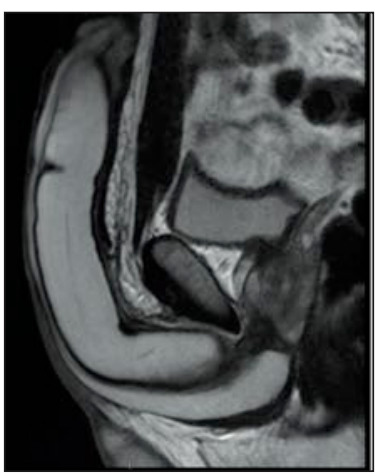

Figure 5.

Postoperative sagital MRI (8 months post-operativelly) showing a thickening of the tunica albuginea of the left cavernosal body.

ies injury was found (Figure 1). A 18 Ch urethral catheter was inserted. Defect of both two cavernosal bodies were repaired by 4-0 Prolene stiches and a end-to-end anastomosis of the urethra was performed with tensionfree 5-0 Vicryl double running sutures (Figure 2).

A broad spectrum antibiotic (cefuroxime $2 \mathrm{~g}$ ) was given during hospital stay. The patient was dismissed 2 days after surgical intervention with $18 \mathrm{Ch}$ Foley catheter.

The urethral catheter was removed at the 12-th postoperative day after a retrograde urethrography that documented a regular urethral profile without signs of stricture neither fistula (Figure 3).

Four months later the patient referred normal erectile and voiding functions; on physical examination a little scar was palpable on the left side of penis shaft and a slight penile curvature on left side during erection without difficulties during sexual intercourse.

At 6 months follow up the patients was evaluated with uroflowmetry demonstrating a max flow rate of $22 \mathrm{ml} / \mathrm{s}$; validated questionnaires demonstrated optimal functional outcomes (IPSS $=3$, QoL $=0$ and IIEF-5 $=23$ ).

IPSS: International Prostate Symptoms Score

QoL: Quality of life

IIEF-5: International Index of Erectile Function.

Eight months later the patients underwent a magnetic resonance imaging (MRI) of the penis, performed after the injection of $10 \mathrm{mcg}$ of prostaglandin-El in the corpora cavenosa, showing a little curvature on the left side of the penile shaft with thickening of the tunica albuginea (Figures 4, 5). At 12 months follow up penile color Doppler ultrasonography confirmed the presence of a thin scar on the middle third of left cavernosal body, with a minimal curvature. IPSS, QoL and IIEF-5 were repeated with confirmation of the functional outcomes.

\section{Discussion}

Discussion and supplementary tables and references are posted in Supplementary Materials on www.aiua.it

\section{Conclusions}

Penile fracture is an uncommon urological emergency, probably it is an under reported or hidden and it remains a clinical problem for delayed diagnosis or treatment and mismanagement; very rarely it is associated with complete urethral rupture. We present the $23^{\text {rd }}$ case of penile fracture with total urethral disruption reported in Englishwritten literature. In our opinion, in order to prevent long term complications, in case of clinical suspicion of penile fracture, especially if it is associated to urethral disruption, emergency surgery should be the first choice of treatment.

\section{References}

1. Eke N. Fracture of the penis. Br J Surg. 2002; 89:555-65.

2. Martinez Portillo FJ, Seif C, Spahn M, et al. Penile fractures: Controversy of Surgical vs. Conservative Treatment. Aktuel Urol. 2003; 34:33-6.

3. Mydlo JH, Hayyeri M, Macchia RJ. Urethrography and cavernosography imaging in a small series of penile fractures: a comparison with surgical findings. Urology 998; 51:616-619.

\section{Correspondence}

Marco Garofalo, MD - marco.garofalo@unibo.it

Lorenzo Bianchi, MD - lorenzo.bianchi3@gmail.com

Giorgio Gentile, MD (Corresponding Author) - dr.giorgio.gentile@gmail.com

Valerio Vagnoni, MD - vagno07@libero.it

Marco Borghesi, MD - mark.borghesil@gmail.com

Riccardo Schiavina, MD - rschiavina@yahoo.it

Daniele Romagnoli MD - danieleromagnoli@hotmail.it

Eugenio Brunocilla MD - eugenio.brunocilla@unibo.it

Department of Urology, University of Bologna, S.Orsola-Malpighi Hospital

Alessandro Franceschelli, MD - alessandro.franceschelli@aosp.bo.it

Fulvio Colombo, MD - fulvio.colombo@aosp.bo.it

Andrology Unit, Sant'Orsola-Malpighi Hospital

Beniamino Corcioni, MD - beniamino.corcioni@aosp.bo.it

Rita Golfieri, MD - Italyrita.golfieri@aosp.bo.it

Department of Radiology, University of Bologna, S.Orsola-Malpighi Hospital, Bologna, Italy, Via P. Palagi, 9 - 40138 Bologna, Italy 